\title{
The base of the tongue regulates the main occluding area in the patients with mandibular prognathism
}

\author{
Yuko Yamada ${ }^{1}$, Hiroyuki Kanzaki ${ }^{2}$, Satoshi Wada ${ }^{3}$, Makoto Kurokawa ${ }^{4}$, Hideho Handa ${ }^{5}$, Hitoshi Kato ${ }^{6}$, Kazutoshi Nakaoka ${ }^{7}$, Yoshiki \\ Hamada $^{8}$, Yoshiki Nakamura ${ }^{9}$ and Hiroshi Tomonari ${ }^{10}$ \\ ${ }^{1}$ Research Assistant, Department of Orthodontics, School of Dental Medicine, Tsurumi University, Yokohama, Japan \\ ${ }^{2}$ Associate Professor, Department of Orthodontics, School of Dental Medicine, Tsurumi University, Yokohama, Japan \\ ${ }^{3}$ Assistant Professor, Department of Orthodontics, School of Dental Medicine, Tsurumi University, Yokohama, Japan \\ ${ }^{4}$ Research Fellow, Department of Orthodontics, School of Dental Medicine, Tsurumi University, Yokohama, Japan \\ ${ }^{5}$ Private Practice, Department of Orthodontics, Handa Orthodontic Office, Yamato, Japan \\ ${ }^{6}$ Private Practice, Dental Clinic of Tokyo Securities Industry Health Insurance Society, Tokyo, Japan \\ ${ }^{7}$ Lecture, Department of Oral and Maxillofacial Surgery, School of Dental Medicine, Tsurumi University, Yokohama, Japan \\ ${ }^{8}$ Professor, Department of Oral and Maxillofacial Surgery, School of Dental Medicine, Tsurumi University, Yokohama, Japan \\ ${ }^{9}$ Research Scientist, Department of Orthodontics, School of Dental Medicine, Tsurumi University, Yokohama, Japan \\ ${ }^{10}$ Professor and Department Chair, Department of Orthodontics, School of Dental Medicine, Tsurumi University, Yokohama, Japan
}

\begin{abstract}
Objective: We previously reported that the main occluding area is more stable on the mandibular first molar than on the maxilla in the patients with mandibular prognathism, which signifying that the anatomical structure that be moved backward with mandibular setback would regulate the position of the main occluding area. Therefore, we analysed the anatomical structure which regulates the orientation of the main occluding area by using the cross-sectional data of the location of the main occluding area and cephalometric maxillo-facial structures before and after surgical orthodontic treatment.
\end{abstract}

Materials and methods: 14 patients with mandibular prognathism and 14 patients with mandibular prognathism who had undergone orthognathic surgery participated in this study. The main occluding area was identified by clenching of Temporary Stopping. Each cephalometric landmarks were projected to the occlusal plane, and the distance from the main occluding area to each projected cephalometric landmarks were measured and were compared before and after surgery.

Results: The change in the distance from the main occluding area to the point of intersection of the base of the tongue and mandibular plane before and after surgical orthodontic treatment was the smallest among all reference points, such as Keyridge, Ptm, and Me, with stastical significance.

Conclusion: The main occluding area in the patients with mandibular prognathism is stable in the mandibular first molar, and its position would be regulated by the base of the tongue.

\section{Introduction}

The main occluding area is defined as the occlusal area where hard food is initially crushed in the early step of mastication $[1,2]$. The main occluding area plays a central role in hard food crushing during mastication, and is an essential factor for both mastication and occlusion [3]. Kato et al reported that the main occluding areas in the subjects with normal occlusion are located within $5 \mathrm{~mm}$ of range between the mesiolingual ridge of the maxillary first molar and distobuccal cusp tip of the mandibular first molar [3]. Regarding the main occluding area in the patients with mandibular prognathism (MP), we reported that the main occluding area also exist in these patients, and it was stable on the first molar even after orthognathic surgery in the mandible, though it was changed from the second premolar to the first molar in the maxilla [4]. We had presumed that the main occluding area is preferentially determined in the mandible rather than in the maxilla in the patients with MP. Orthognathic surgery for the patients with MP setback the mandible, therefore it is possible that the structure which is setback along with the mandible would regulate the main occluding area.
As to the mechanism for orientation of the main occluding area, Kato et al. reported that both the sensation of periodontal membrane and the coordinated movements of tongue and cheek at clenching regulate the position of main occluding area [2]. Among them, the tongue transports a food bolus from the masticatory area to the area and helps mastication by crushing food between the palate and the dorsum of the tongue $[5,6]$. A food bolus is firstly collected on the dorsum of tongue, then the anterior part of the tongue shifted to the chewing side, and the dorsum of tongue is pressed on the lingual side

${ }^{\star}$ Correspondence to: Hiroyuki Kanzaki, Department of orthodontics, School of Dental Medicine, Tsurumi University, 2-1-3 Tsurumi, Tsurumi-ku, Yokohama, Kanagawa pref., 230-8501, Japan, Tel: +81-45-580-8507; Fax: +81-45-573-9599; E-mail: kanzaki-h@tsurumi-u.ac.jp

Key words: main occluding area, base of tongue, mandibular prognathism, orthognathic surgery, lateral cephalometric radiograph, cephalometric landmarks

Received: December 21, 2020; Accepted: January 07, 2021; Published: January 11,2021 
of tooth, which makes the food bolus delivered on the occlusal surface [7]. Thus, the tongue plays an important role in carrying a food bolus on the occlusal surface.

Kato et al. also described that the occlusal contact area and the occlusal contact point where occlusal force is applied in the direction of the perpendicular to the occlusal plane regulate the orientation of the main occluding area in the subject with normal occlusion $[2,8]$. However, no study has yet examined how the main occluding area is regulated in the patients with MP, especially after orthognathic surgery. The objective of this study was to identify the anatomical structure which regulates the orientation of the main occluding area by analyzing the cross-sectional data of the location of the main occluding area and cephalometric maxilla-facial structure before and after surgical orthodontic treatment.

\section{Materials and methods}

This study is a cross-sectional study analyzing the patients with MP before and after surgical orthodontic treatment.

Prior to this study, the experimental protocol was approved by the Ethics Committee of Tsurumi University, School of Dental Medicine (Approved No.934 and 1808) and conformed to the principles of the Declaration of Helsinki. Written informed consent was obtained for all patients before this study. Furthermore, the principles of the Declaration of Helsinki was conformed during experiment. All patients provided informed consent, and written consent forms were obtained before this study.

Patients with MP who had undergone orthognathic surgery (MPOS) were included in this study.

The MP group, comprising 14 patients with MP who required orthognathic surgery at the Department of Orthodontics, School of Dental Medicine, Tsurumi University, met the following inclusion criteria:

- no congenital abnormalities,

- no missing teeth other than the third molar,

- no major restoration covering the cusp,

- overbite of $1.0 \mathrm{~mm}$ or more,

- overjet of less than $0 \mathrm{~mm}$,

- no notable mandibular asymmetry,

- severe Angle Class III molar relationship with the occlusion between the maxillary second premolar and mandibular first molar , and

- skeletal Class III intermaxillary relationship in ANB angle and Wits analysis.

The MPOS group, consisting of 14 patients with MP who had undergone orthognathic surgery, met the following inclusion criteria:

- no missing teeth other than the third molar at the first visit,

- no major restoration covering the cusp,

- appropriate overjet and overbite,

- midline deviation of $1.0 \mathrm{~mm}$ or less,

- preoperative occlusion between the maxillary second premolar and mandibular first molar,
- at least 1 year since orthognathic surgery,

- Angle Class II molar relationship due to the extraction of the maxillary bilateral first premolars in orthodontic treatment and mandibular setback.

- normal intermaxillary relationship identified or classified by ANB angle and Wits analysis.

\section{Identification of the main occluding area}

The method for identifying the main occluding area was described elsewhere [4].

Briefly, one piece of dental stopping was placed on the center of the dorsal surface of the tongue as test food, and the subject clenched it once on the occluding area that was used during mastication. The location of the dental stopping on the dental arch was defined as the main occluding area (Figure 1).

\section{Evaluation of the main occluding area using lateral cephalometric radiographs}

Lateral cephalograms were taken before and after orthodontic treatment with the teeth in occlusion and the lips in a relaxed position. The radiographs were traced and recorded their main occluding area on dental cast.

To examine the distance from the main occluding area to the point of each anatomical structure, the landmarks used for this study are shown in Figure 2; 1) the most anterior point of lower lip, 2) Pogonion $(\mathrm{Pog}), 3)$ the edge of upper central incisor, 4) the tip of the tongue, 5) anterior nasal spine (ANS), 6) Menton (Me), 7) inside of symphysis, 8)Orbitale (Or), 9) keyridge, 10) the most anterior point of the hyoid bone, 11) Pterygomaxillare (Ptm), 12) the most anterior point of tip of the soft palate, 13) the most protruding point of the root of tongue

a)

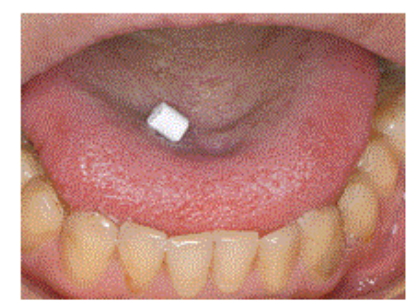

b)

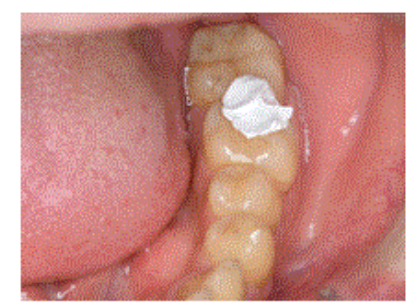

Figure 1. Identification of the main occluding area using a dental stopping a) Placement of the stopping on the tongue.

b) After chew the stopping, indicating the main occluding area 
$[9,10], 14)$ the top of the epiglottis, 15) the intersection point of the posterior pharyngeal wall and mandibular plane.

Each cephalometric landmarks were projected to the occlusal plane, and the distance from the main occluding area to each projected cephalometric landmarks were measured and were compared before and after surgery (Figure 2). Mesial direction from the main occluding area is determined as positive value, and distal direction from the main occluding area is determined as negative value.

All measurements of distances were repeated three times and their average were compared between MP and MPOS. The measurement error was calculated by intraclass-reliability (ICC). The values of ICC were between 0.9 and 1.0 in this study.

\section{Power analysis}

Power analysis was performed using $\mathrm{G}^{\star}$-power. The reguired total sample size was 28 , under the following parameters; Effect size $=0.9$, significance level $=0.05$, power $=0.7$.

\section{Statistical analysis}

The statistical analysis was performed using SPSS statistical software (SPSS Japan, Tokyo, Japan). After calculating the average and standard error, normal distribution of all data was confirmed (Shapiro-Wilk).

The difference of the distance from the main occluding area before and after orthodontic treatment were determined by the paired $t$-test. Statistical significance was defined as the $P$ value $<0.05$. Multiple comparison among three parameters was performed by $t$-test with Bonferroni correction. Statistical significance was defined as the $P$ value $<0.05 / 3$.

\section{Results}

Location of the main occluding area before and after orthodontic treatment

The change in the distance from the main occluding area to the most protruding point of the root of tongue before and after orthodontic treatment was the smallest among all reference points, followed by the tip of the soft palate, tongue tip, Or, upper central incisal edge, the most anterior point of lower lip, Ptm, inside of symphysis, Pog, ANS, the hyoid anterior protruding point of the hyoid bone, Me, keyridge, mandibular plane and top of the epiglottis and the point of intersection posterior pharyngeal wall (Table 1). The change of the distance from the main occluding area to the structure that move less after orthognathic surgery, such as keyridge, Ptm, Me, mandibular plane and top of the epiglottis and the point of intersection posterior pharyngeal wall and the hyoid anterior protruding point of the hyoid bone was significantly larger than that to the base of the tongue. These results indicated that the base of tongue seemed to closely relate to the change of the position of the main occluding area.

\section{The change of the location of the main occluding area is closely relate to that of the base of the tongue}

We then statistically analyzed of which anatomical structure is most closely relate to the change of the main occluding area (Figure 3 ). Three representative reference points, the base of the tongue, maxilla, and mandible were chosen. The base of the tongue exhibited the smallest value as compared to keyridge and Me. These results indicated that the change of the location of the main occluding area would closely relate to the change of the position of the base of the tongue, among anatomical structures.

\section{Discussion}

We discovered that the change in the distance from the base of the tongue to the main occluding area before and after the surgical orthodontic treatment was the smallest among representative anatomical structures. Our results suggest that the base of the tongue regulates the position of the main occluding area in the patients with MP.

Angle established the method to classify malocclusion based on the mesiodistal positional relationship with the occlusion between the maxillary and mandibular first molar [11]. The Angle classification is on the assumption that maxillary first molar is unchanging in its position. In later year, Atkinson and his colleagues demonstrated that the line connected the left and right mesial roots of the maxillary first molar were matched to keyridge by using a number of skulls [12]. In addition, the line of action of the occlusal force started from first molars and passed through keyridge [13]. Furthermore, keyridge is the area where the strong occlusal force applied on the maxillary first molar

Table 1. Distance of main occluding area from each reference point in patients with mandibular prognathism before and after surgical orthodontic treatment

\begin{tabular}{|c|c|c|c|c|c|c|}
\hline & \multicolumn{2}{|c|}{ Before(N=14) } & \multicolumn{2}{|c|}{ After(N=14) } & \multicolumn{2}{|c|}{ Change } \\
\hline & mean & S.E. & mean & S.E. & mean & S.E. \\
\hline 1. most prominent point of lower lip & 50.6 & 1.9 & 46.9 & 1.0 & 3.7 & 0.7 \\
\hline 2. Pog & 36.2 & 2.2 & 32.0 & 0.9 & 4.2 & 0.7 \\
\hline 3. upper central incisal edge & 31.0 & 1.6 & 34.5 & 0.7 & 3.5 & 0.6 \\
\hline 4. tongue tip & 26.9 & 1.8 & 24.4 & 0.8 & 2.5 & 0.8 \\
\hline 5. ANS & 24.4 & 1.4 & 28.7 & 0.9 & 4.3 & 0.8 \\
\hline 6. Me & 28.6 & 3.1 & 23.5 & 1.1 & 5.1 & 0.9 \\
\hline 7. inside border of symphysis & 22.6 & 2.4 & 18.4 & 0.9 & 4.2 & 0.7 \\
\hline 8. Or & 5.3 & 1.2 & 8.5 & 0.9 & 3.2 & 0.6 \\
\hline 9. keyridge & -4.2 & 1.4 & 1.0 & 0.9 & 5.2 & 0.5 \\
\hline 10. the anterior protruding point of the hyoid bone & -22.0 & 2.8 & -17.0 & 2.1 & 5.0 & 1.7 \\
\hline 11. Ptm & -31.0 & 1.2 & -27.0 & 0.8 & 4.0 & 0.7 \\
\hline 12. tip of the soft palate & -42.0 & 1.8 & -39.7 & 0.8 & 2.3 & 0.6 \\
\hline 13. the most protruding point of the root of tongue & -42.0 & 2.0 & -40.8 & 0.8 & 1.2 & 0.4 \\
\hline 14. top of the epiglottis & -49.9 & 2.4 & -44.1 & 1.2 & 5.8 & 0.8 \\
\hline 15. intersection of posterior pharyngealwall and mandibular plane & -58.5 & 1.8 & -51.9 & 1.4 & 6.6 & 1.2 \\
\hline
\end{tabular}




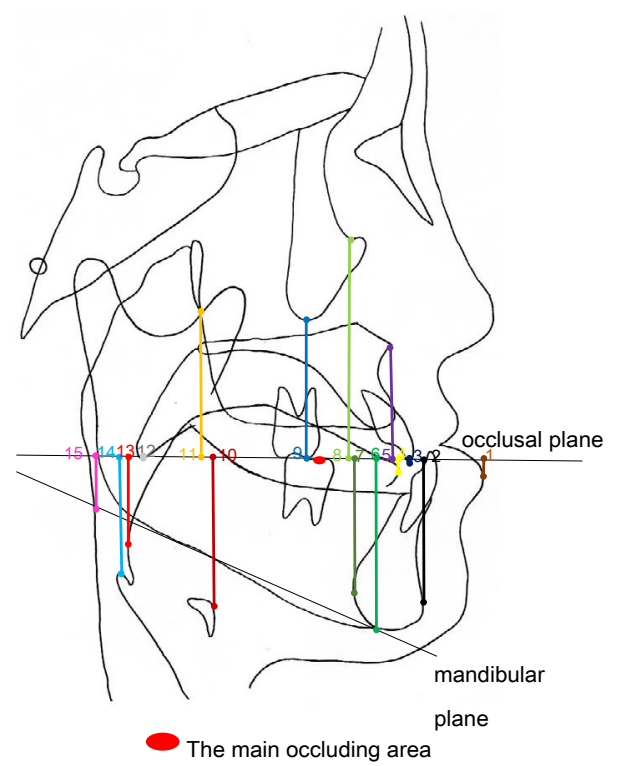

Figure 2. The distance from the main occluding area to the point of each anatomical structure

The foot of a perpendicular from each cephalometric landmarks to the occlusal plane were used for the reference point. 1) most prominent point of lower lip, 2) Pog,3) upper central incisor edge, 4) tongue tip, 5) ANS, 6) Me, 7) inside border of symphysis, 8) Or, 9) keyridge, 10) the anterior protruding point of the hyoid bone, 11) Ptm, 12) tip of the soft palate, 13) the most protruding point of the root of tongue, 14) top of the epiglottis, 15) intersection of posterior pharyngeal wall and mandibular plane

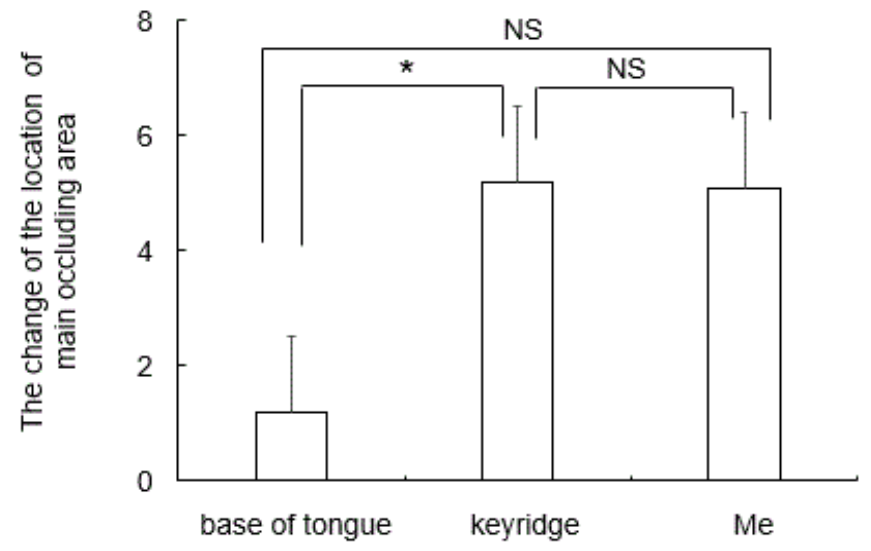

Figure 3. The change of the location of the main occluding area before and after orthodontic treatment

The change of the location of the main occluding area before and after orthodontic treatment are shown. ${ }^{*} P<0.05$ between groups. NS indicates no significant difference between groups

is distributed superiorly and posteriorly [14]. Together, it has been thought that keyridge plays the central role in occlusion. The main occluding area is related to the keyridge.

As to the factors which regulate the position of the main occluding area on the mandibular dental arch, the line of action of the occlusal force applied in the mandibular first molar is distributed to the center of the ridge of the distobuccalor distal cusp that goes through the occlusal surface, and matched to the main occluding area [15]. The main occluding area in the subjects with normal occlusion are located distobuccal cusp tip of the mandibular first molar $[3,16]$. In addition, the main occluding area in the patients with MP is stable on the mandibular first molar after orthognathic surgery [4].
Our results indicated that the base of the tongue was the smallest change in the distance from the main occluding area before and after the surgical orthodontic treatment, and the change in the distance from the main occluding area to keyridge was significantly larger than that from the base of the tongue. Furthermore, the change in the distance from the main occluding area to the structure that moved less after orthognathic surgery, such as Ptm, was significantly larger than that to the base of the tongue. These results suggest that the base of the tongue is related to the regulation of the location of the main occluding area before and after orthodontic treatment. This study is a cross-sectional study, but it is necessary to compare it in a longitudinal study in the future.

The tongue plays the variety of roles such as collecting a food bolus and delivering them on the occlusal surface during the mastication [8]. A food bolus is carried by tongue to the molar region where the main occluding area exist, and subsequently the chewing starts [17]. Muscles, such as the genioglossus muscle and the superior and inferior longitudinal muscle of tongue attached to the base of the tongue, and these muscles control the antero-posterior movement of the tongue, which makes possible to play an important role not only on mastication but also on swallowing and articulatory function [18].

There are many reports for the change of the cranio-facial structure after surgical orthodontic treatment. The posterior pharyngeal wall goes backward slightly by mandibular setback and is generally stable during retention [19]. The width of the airway decreases after mandibular setback in the area from the intermediate part of the soft palate to the posterior border of the base of the tongue, though there is no significant difference in the decrease of that in the area of the upper part the soft palate and the epiglottic vallecular [20]. The airway was decreased 2.24 $\mathrm{mm}$ around the uvula, $2.5 \mathrm{~mm}$ at the angle of the mandible, and 1.05 $\mathrm{mm}$ around the epiglottis after mandibular setback more than $8 \mathrm{~mm}$ [21]. As for the epiglottis, it is reported that the swallowing triangle, that composed of the top of the epiglottis, the posterior pharynx, and hyoid bone, moves postero-inferiorly, just after mandibular setback, though it returns at certain extent overtime [22]. Together, the change of the position of the base of the tongue is generally variable, and which influence the stability of the mandible after surgical orthodontic treatment. Our results revealed some difference between the change of the position of the main occluding area and that of the base of tongue though this was smallest as compare to the other anatomical structures. We presumed that this difference is due to the elastic deformation of the tongue.

\section{Conclusions}

The main occluding area in the patients with MP is stable in the mandibular first molar, and its position would be regulated by the base of the tongue.

\section{Disclosure of interest}

The authors report no conflict of interest.

\section{Funding information}

This research did not receive any specific grant.

\section{Informed consent statement}

Written informed consent were obtained from all participants. 


\section{Ethical approval statement}

This study was approved by the Ethics Committee of Tsurumi University School of Dental Medicine (Approved No.934 and 1808).

\section{References}

1. Kato H, Tanaka Y, Nozawa K, Miura H, Hasegawa S (2001) Observation of Intraoral Food Flow in Mastication. J Jpn Soc Stomatognath Funct 7:81-89.

2. Kato H (2010) Practical occlusion theory based on the main occluding area. Tokyo: Dental Diamond.

3. Nishinaka H (2011) Significance of the main occluding area in prosthetic treatment. Shikoku Dental Research 24:1-15.

4. Kurokawa M, Kanzaki H, Tokiwa H, Handa H, Nakaoka K, et al. (2016) The main occluding area in normal occlusion and mandibular prognathism. Angle Orthodontist $86: 87-93$

5. Kawamura Y (1966) Oral Physiology for dentist students. $4^{\text {th }}$ ed. Tokyo: Nagasueshoten.

6. Kido T (1996) The Study of Food Behavior on Mastication. J Jpn Prosthodont Soc 40:524-534

7. Izumi H, Asanuma N (2010) Physiology and oral physiology. $3^{\text {st }}$ edition. Tokyo: Gakkenshoin Ltd.

8. Kato H, Furuki Y, Hasegawa S (1996) Observations on the main occluding area in mastication. J Jpn Soc Stomatognath Funct 2:119-127.

9. Pushkar Mehra, Michael Doenie, Maecos C. Pita, Larry M. Wolford (2001) Pharyngeal airway space changes after counterclockwise rotation of the maxillomandibular complex. AJODO 120(2):154-159.

10. Goncalves JR, Buschbang PH, Goncalves DG, Wolford LM (2006) Postsurgical stability of oropharyngeal airway changes following counterclockwise maxillomandibular advancement surgery. J oral Maxillofac Surg 64:755-762. [Crossref]

11. Angle EH (1899) Classification of malocclusion. Dental Cosmos 41:248-264.
12. Atkinson SR (1942) Some anatomic factors guiding treatment therapy. American Journal of Orthodontics and Oral Surgery 28:704-720.

13. Endoh R, Satoh C, Hattori Y, Hisamatsu H, Watanabe M (2006) Relationship between occlusal force in dental arch and facial morphology. J Jpn Soc Stomatognath Funct 12:136-137.

14. Kaimoto K, Tamatsu Y, Ide Y (2000) A Measurement of Local Elastic Modulus of Buccal Compact Bone of Human Facial Cranium. Jpn J Oral Biol 42:213-224.

15. Hattori Y (2013) Occlusal Morphology as a Reflection of Oral Function. Ann Jpn Prosthodont Soc 5:14-18.

16. Tokuda A, Kato H, Miura H, Okada D, Hoshino K, Hasegawa S (2006) The main occluding area in consideration of the occlusal relation. J Jpn Soc Stomatognath Funct $13: 31-37$.

17. Fujii H (1971) Influence of masticatory movements of tongue on the food pulverization. Jap J Oral Biol 13:485-498.

18. Takehara S, Shimoyama K (2006) Anatomical Structure and Functional Training of Tongue. J J Gerodont 21:44-47.

19. Emata K, Mitani H, Sakamoto T (1983) Effect of orthognathic surgery on skeleta mandibular prognathism. Changes of the tissue profile, pharyngeal air way and hyoid position. J Jpn Orthod Soc 42:69-84. [Crossref]

20. Terakado K, Shiina Y, Naruse T, Sueishi K, Suzuki T, Yamaguchi H, et al. (1997) Effects of Surgical Orthodontic Treament on Oral Cavity, Tongue, and Pharyngeal AirwayPart1: Changes caused by mandibular setback surgery. The Journal of the Tokyo Dental College Society 97:181-191.

21. Suzuki M, Uzuka S, Watanabe N, Miyashita W, Fujishiro T, Shoji H, et al. (2015) Jpn J Jaw Deform 25:201-206.

22. Oya T, Matsumoto N (2013) Stability of the swallow-triangle on lateral cephalograms in skeletal ClassIII cases treated by mandibular setback surgery. J Osaka Odontol Soc 76:9-17.

Copyright: (C2021 Yamada Y. This is an open-access article distributed under the terms of the Creative Commons Attribution License, which permits unrestricted use, distribution, and reproduction in any medium, provided the original author and source are credited. 